\title{
Mechanisms for Electron Uptake by Methanosarcina acetivorans during Direct Interspecies Electron Transfer
}

\author{
(D)Dawn E. Holmes, ${ }^{\mathrm{a}, \mathrm{b}}$ Jinjie Zhou, ${ }^{\mathrm{a}, \mathrm{c}}$ (D) Toshiyuki Ueki, ${ }^{\mathrm{a}}$ Trevor Woodard, ${ }^{\mathrm{a}}$ (D) Derek R. Lovley ${ }^{\mathrm{a}}$ \\ aDepartment of Microbiology, University of Massachusetts-Amherst, Amherst, Massachusetts, USA \\ bDepartment of Physical and Biological Science, Western New England University, Springfield, Massachusetts, USA \\ Institute for Advanced Study, Shenzhen University, Shenzhen, Guangdong, China
}

Dawn E. Holmes and Jinjie Zhou contributed equally to this work. Author order was determined alphabetically.

ABSTRACT Direct interspecies electron transfer (DIET) between bacteria and methanogenic archaea appears to be an important syntrophy in both natural and engineered methanogenic environments. However, the electrical connections on the outer surface of methanogens and the subsequent processing of electrons for carbon dioxide reduction to methane are poorly understood. Here, we report that the genetically tractable methanogen Methanosarcina acetivorans can grow via DIET in coculture with Geobacter metallireducens serving as the electron-donating partner. Comparison of gene expression patterns in M. acetivorans grown in coculture versus pure-culture growth on acetate revealed that transcripts for the outer-surface multiheme c-type cytochrome MmcA were higher during DIET-based growth. Deletion of $m m c A$ inhibited DIET. The high aromatic amino acid content of $M$. acetivorans archaellins suggests that they might assemble into electrically conductive archaella. $A$ mutant that could not express archaella was deficient in DIET. However, this mutant grew in DIET-based coculture as well as the archaellum-expressing parental strain in the presence of granular activated carbon, which was previously shown to serve as a substitute for electrically conductive pili as a conduit for long-range interspecies electron transfer in other DIET-based cocultures. Transcriptomic data suggesting that the membrane-bound Rnf, Fpo, and HdrED complexes also play a role in DIET were incorporated into a charge-balanced model illustrating how electrons entering the cell through MmcA can yield energy to support growth from carbon dioxide reduction. The results are the first genetics-based functional demonstration of likely outersurface electrical contacts for DIET in a methanogen.

IMPORTANCE The conversion of organic matter to methane plays an important role in the global carbon cycle and is an effective strategy for converting wastes to a useful biofuel. The reduction of carbon dioxide to methane accounts for approximately a third of the methane produced in anaerobic soils and sediments as well as waste digesters. Potential electron donors for carbon dioxide reduction are $\mathrm{H}_{2}$ or electrons derived from direct interspecies electron transfer (DIET) between bacteria and methanogens. Elucidating the relative importance of these electron donors has been difficult due to a lack of information on the electrical connections on the outer surfaces of methanogens and how they process the electrons received from DIET. Transcriptomic patterns and gene deletion phenotypes reported here provide insight into how a group of Methanosarcina organisms that play an important role in methane production in soils and sediments participate in DIET.

KEYWORDS anaerobic respiration, extracellular electron transfer, Methanosarcina, Geobacter, direct interspecies electron transfer, DIET, Rnf complex, c-type cytochrome, methanogen, archaea
Citation Holmes DE, Zhou J, Ueki T, Woodard T, Lovley DR. 2021. Mechanisms for electron uptake by Methanosarcina acetivorans during direct interspecies electron transfer. mBio 12: e02344-21. https://doi.org/10.1128/mBio .02344-21.

Editor Eleftherios T. Papoutsakis, University of Delaware

Copyright $\odot 2021$ Holmes et al. This is an open-access article distributed under the terms of the Creative Commons Attribution 4.0 International license. Address correspondence to Dawn E. Holmes, dholmes@wne.edu.

Received 6 August 2021

Accepted 31 August 2021

Published 5 October 2021 
he pathways for carbon and electron flux in methanogenic environments are of interest because of the biogeochemical significance of methane production in diverse soils and sediments as well as the importance of anaerobic digestion as a bioenergy strategy $(1,2)$. Diverse communities of bacteria convert complex organic matter primarily to acetate and carbon dioxide, which are then converted by methanogenic archaea to methane. Low-potential electrons derived from the oxidation of organic compounds to acetate and carbon dioxide are delivered from the bacterial community to methanogens to provide the necessary reducing power for the reduction of carbon dioxide to methane.

Two fundamentally different mechanisms for this interspecies electron transfer are known. In direct interspecies electron transfer (DIET), electron-donating microbes and methanogens establish direct electrical connections that enable electron transfer from the electron-donating partner to the methanogen to support carbon dioxide reduction (3-5). In interspecies $\mathrm{H}_{2}$ transfer, the electron-donating partner transfers electrons to protons, generating $\mathrm{H}_{2}$, which functions as a diffusive electron carrier to $\mathrm{H}_{2}$-utilizing methanogens, which oxidize the $\mathrm{H}_{2}$ to harvest electrons for carbon dioxide reduction (6-8). Formate can also serve as a substitute for $\mathrm{H}_{2}(7,9,10)$.

The relative importance of DIET and interspecies $\mathrm{H}_{2}$ /formate transfer in methanogenic soils/sediments or most anaerobic digesters is unknown. Measurements of $\mathrm{H}_{2}$ turnover rates in methanogenic soils, sediments, and anaerobic digesters accounted for less than $10 \%$ of the electron flux required for the observed rates of carbon dioxide reduction to methane (11-13), suggesting that $\mathrm{H}_{2}$ exchange was not the primary route for interspecies electron transfer (2). Those results do not rule out interspecies formate exchange, but rapid exchange between formate and $\mathrm{H}_{2}$ /carbon dioxide in methanogenic environments prevents accurate assessment of formate fluxes (14). The relatively low reported rates of $\mathrm{H}_{2}$ turnover are consistent with DIET, but a method for directly measuring the electron fluxes between cells in complex environments has not yet been developed.

An alternative strategy for elucidating the significance of interspecies $\mathrm{H}_{2}$ /formate transfer and DIET might be to extrapolate from the composition of the microbial community and transcriptional or proteomic data (15-17). For example, in situ gene expression patterns of Geobacter and Methanothrix species, which were abundant in methanogenic rice paddy soils, suggested that they participated in DIET (17). The likely participation of paddy-soil Geobacter species in DIET could be surmised from high levels of expression for electrically conductive pili (e-pili) and a c-type cytochrome known to be important for DIET. High expression of genes for carbon dioxide reduction in Methanothrix species, which are unable to use $\mathrm{H}_{2}$ or formate as an electron donor, indicated that Methanothrix species were one of the electron-accepting partners for DIET. However, such analyses are far from comprehensive, in part because the full diversity of microbes that can participate in DIET is poorly understood. New genera of bacteria and methanogens capable of DIET are increasingly being identified (18-20). Furthermore, gene expression patterns diagnostic for ongoing DIET need to be elucidated for microorganisms, such as Syntrophus (18) and some Methanosarcina species (16, 19, 21-23), that have the physiological flexibility to participate in either DIET or interspecies $\mathrm{H}_{2}$ /formate transfer.

Comparative transcriptomic analysis of Methanosarcina barkeri growing via DIET versus interspecies $\mathrm{H}_{2}$ transfer revealed potential routes for intracellular electron flux for DIET that employ protein complexes and electron carriers that are also important for the conversion of carbon dioxide to methane with $\mathrm{H}_{2}$ as the electron donor (21). Outer-surface electrical contacts for DIET were not definitively identified. M. barkeri lacks multiheme outer-surface c-type cytochromes (24), which are important electrical contacts for extracellular electron exchange in some bacteria and archaea (2). Methanosarcina mazei, which, like M. barkeri, can reduce carbon dioxide with electrons derived from $\mathrm{H}_{2}$ or DIET, has a gene for a five-heme c-type cytochrome, but deletion of the cytochrome gene did not negatively impact DIET (19). 


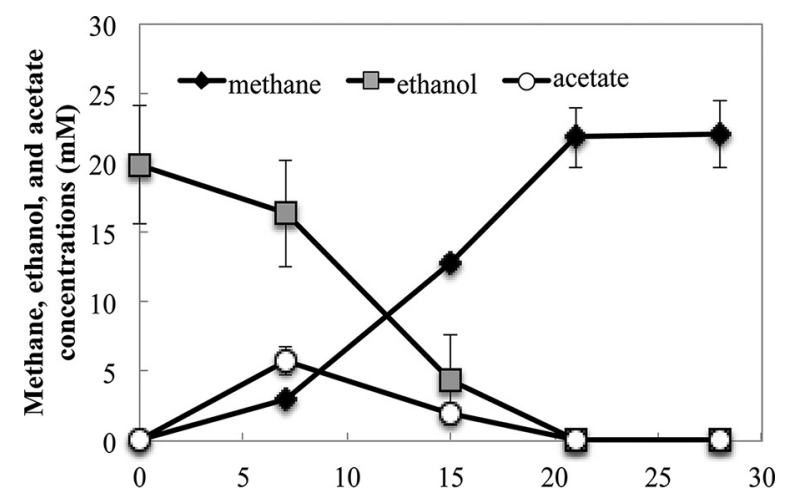

FIG 1 Ethanol consumption and production of methane and acetate in cocultures established with G. metallireducens and M. acetivorans after four successive transfers. Data are means and standard deviations for triplicate cultures.

M. barkeri and M. mazei are physiologically classified as type I Methanosarcina species (22). Key physiological characteristics of type I Methanosarcina are the ability to use $\mathrm{H}_{2}$ as an electron donor for carbon dioxide reduction as well as for energy conservation from the conversion of acetate to methane via intracellular $\mathrm{H}_{2}$ cycling. Although type I Methanosarcina can serve as the electron-accepting partner for DIET, they are typically most abundant in high-energy environments with relatively high rates of organic carbon turnover in which $\mathrm{H}_{2}$ is more likely to be an intermediate in interspecies electron transfer (22).

In contrast, type II Methanosarcina predominate in more stable, steady-state environments with lower rates of organic matter metabolism likely to favor DIET (22). Key physiological characteristics of type II Methanosarcina include the inability to use $\mathrm{H}_{2}$ as an electron donor, energy conservation during acetate metabolism via an Rnf complex, and the presence of an outer-surface multiheme $c$-type cytochrome that is important for electron transfer to extracellular electron acceptors (22). The fact that type II Methanosarcina are unable to utilize $\mathrm{H}_{2}$ or formate as an electron donor for carbon dioxide reduction but are able to participate in DIET (22) is expected to simplify the study of their routes for electron flux during DIET.

In order to better understand DIET mechanisms in type II Methanosarcina, we investigated DIET in Methanosarcina acetivorans. M. acetivorans is the best-studied type II Methanosarcina species and is genetically tractable (25-28). Transcriptomic and gene deletion studies (29) demonstrated that its multiheme outer-surface c-type cytochrome MmcA is important for extracellular electron transfer to the humic substance analogue anthraquinone-2,6-disulfonate (AQDS). Here, we report that $M$. acetivorans can function as the electron-accepting partner for DIET and provide insights into mechanisms for electron uptake and energy conservation during DIET-based growth.

\section{RESULTS AND DISCUSSION}

Methanosarcina acetivorans can participate in DIET. Cocultures of Geobacter metallireducens and M. acetivorans metabolized ethanol to methane. As previously observed with cocultures of $G$. metallireducens and other electron-accepting partners $(16,30,31)$, an adaption period of 38 to 45 days was required for substantial methane to be produced in the initial coculture. However, with subsequent transfer, ethanol was converted to methane without a substantial lag (Fig. 1).

After four transfers of the coculture, the methane yield was $1.1 \mathrm{~mol} \mathrm{CH} / \mathrm{mol}$ ethanol. Stoichiometric conversion of ethanol to methane yields $1.5 \mathrm{~mol}$ of methane, but a portion of the carbon and electrons is required for biomass production. The methane yield in the $G$. metallireducens- $M$. acetivorans coculture falls within the range of $0.91 \mathrm{~mol} \mathrm{CH} / \mathrm{mol}$ ethanol to $1.31 \mathrm{~mol} \mathrm{CH}_{4} / \mathrm{mol}$ ethanol, yields which were obtained when $G$. metallireducens was the electron donating partner for cocultures grown with 


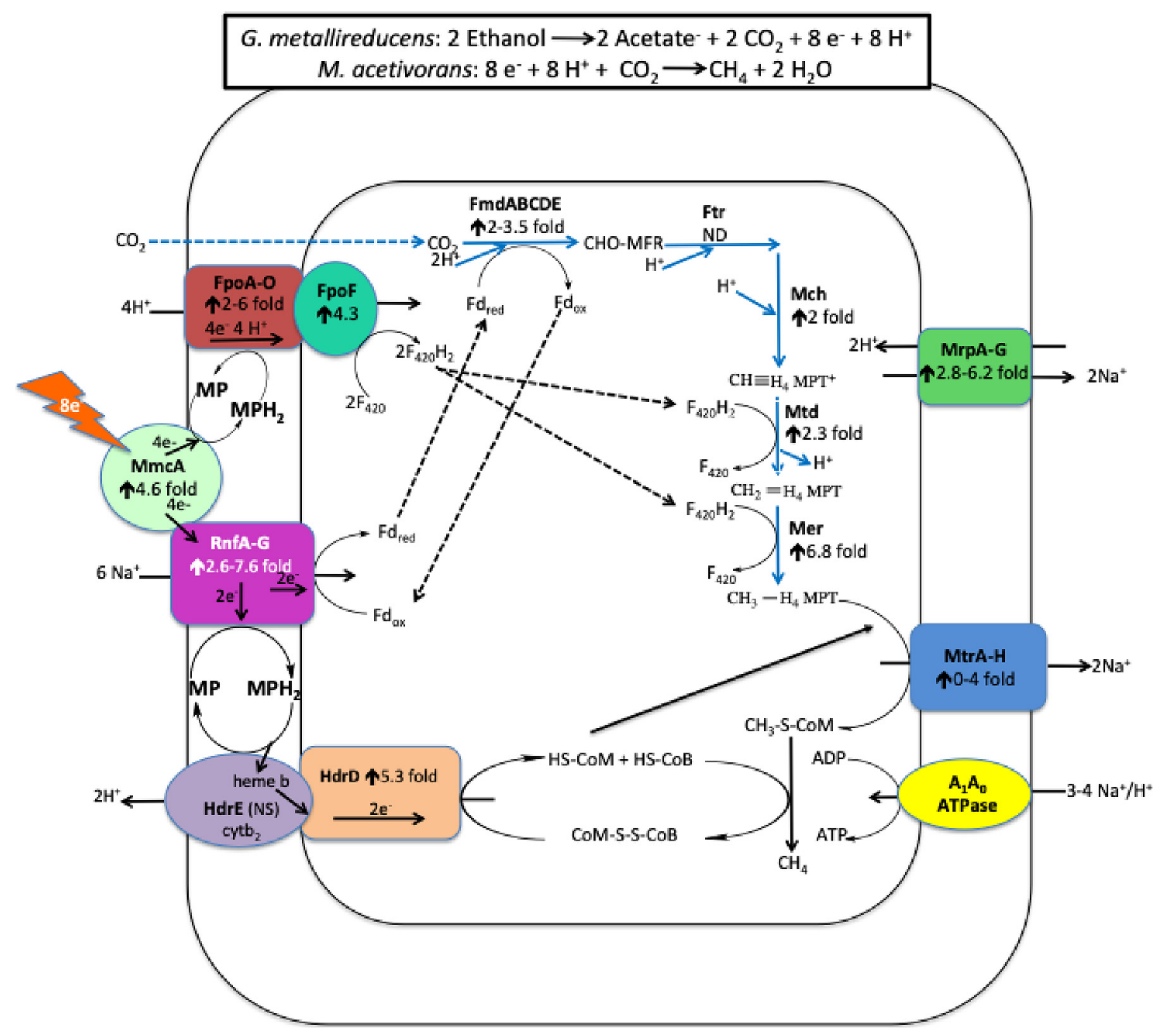

FIG 2 Model for electron and proton flux in Methanosarcina acetivorans during direct interspecies electron transfer (DIET) with Geobacter metallireducens. Ethanol is provided as the source of electrons, and the electron-donating partner (G. metallireducens) transfers those electrons to the electron-accepting partner ( $M$. acetivorans) for carbon dioxide reduction to $\mathrm{CH}_{4}$ through the proposed pathways shown. The degree of increased transcript abundance for subunits of the protein complexes is provided. See the text for a more detailed explanation.

other acetotrophic methanogens, such as Methanothrix harundinacea, M. barkeri, M. mazei, Methanosarcina vacuolata, Methanosarcina horonobensis, and Methanosarcina subterranea $(16,19,22,23,31)$.

Genes for enzymes specific to the carbon dioxide reduction pathway were more highly expressed in M. acetivorans growing in coculture with $G$. metallireducens than in cells growing in pure culture on acetate (Fig. 2; Table S1a). This result is in accordance with the fact that carbon dioxide reduction is required to consume the electrons released from ethanol metabolism, accounting for one-third of the methane produced during DIET. Little or no carbon dioxide reduction is expected during growth solely on acetate. $\mathrm{H}_{2}$ or formate cannot be the interspecies electron carrier between $G$. metallireducens and $M$. acetivorans for carbon dioxide reduction because $G$. metallireducens cannot grow by metabolizing ethanol with the formation of $\mathrm{H}_{2}$ or formate (32), and $M$. acetivorans is unable to use $\mathrm{H}_{2}$ or formate as an electron donor (33).

Quantitative PCR of DNA extracted from the fourth transfer of triplicate cocultures with primers targeting the $16 \mathrm{~S}$ rRNA genes of $G$. metallireducens and M. acetivorans revealed that G. metallireducens accounted for $60 \% \pm 10 \%$ (mean \pm standard deviation) of the cells in the coculture. Confocal and transmission electron microscopy also 


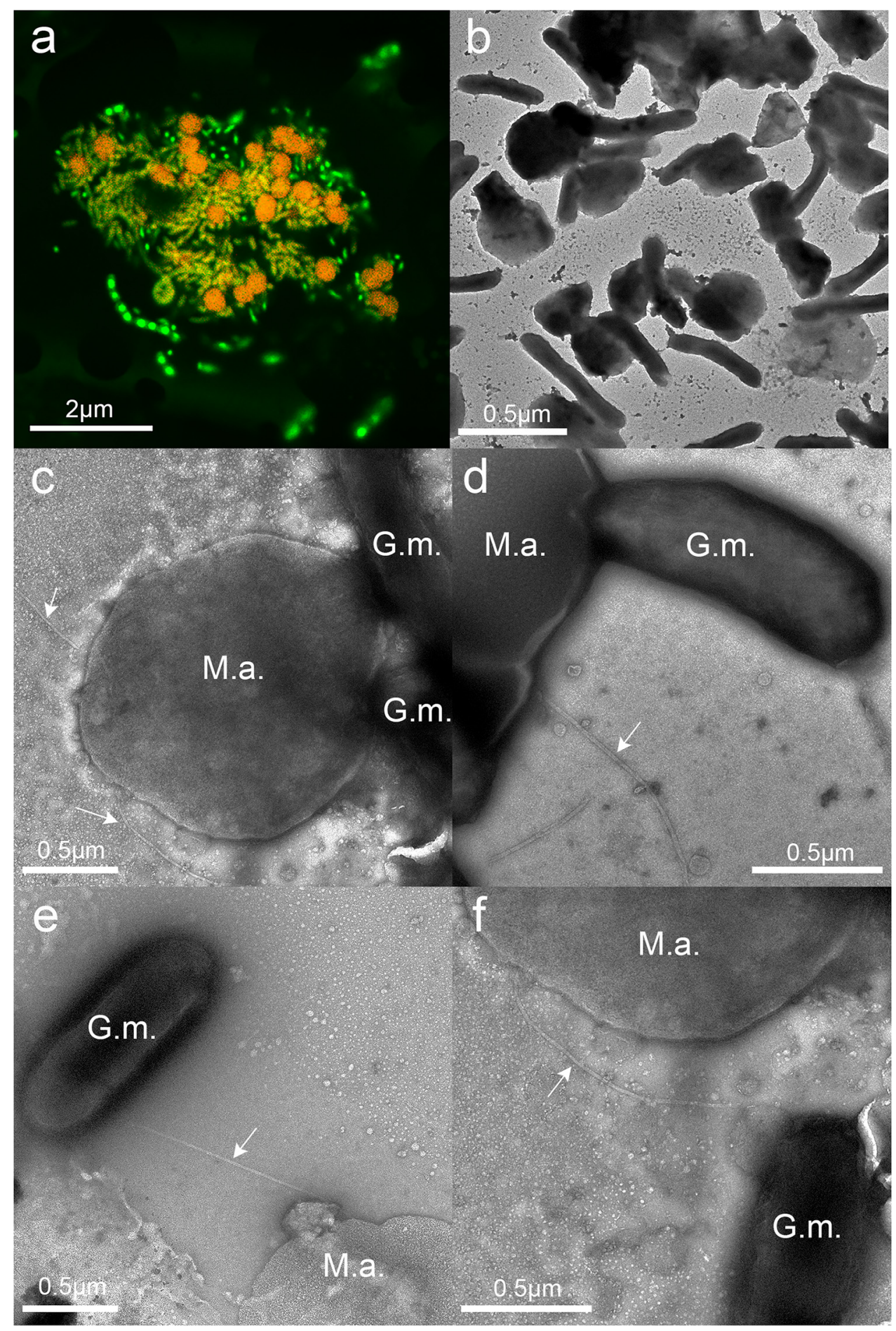

FIG 3 Images of G. metallireducens-M. acetivorans cocultures. (a) Confocal microscopy image demonstrating aggregate size and cell distributions. (b to f) Transmission electron micrographs. Arrows point to archaella extending from the M. acetivorans surface. Abbreviations: M.a., M. acetivorans; G.m., G. metallireducens.

indicated a near-equal abundance of the two species (Fig. 3a and b), and revealed that both species were typically in close proximity, often with more than one cell of each species adjacent to its DIET partner (Fig. 3a and b).

Potential role(s) for the archaellum in DIET. Higher-magnification transmission electron microscopy (TEM) images provided further insights into the interactions between $G$. metallireducens and M. acetivorans (Fig. $3 \mathrm{c}$ to e). The outer surfaces of cells 

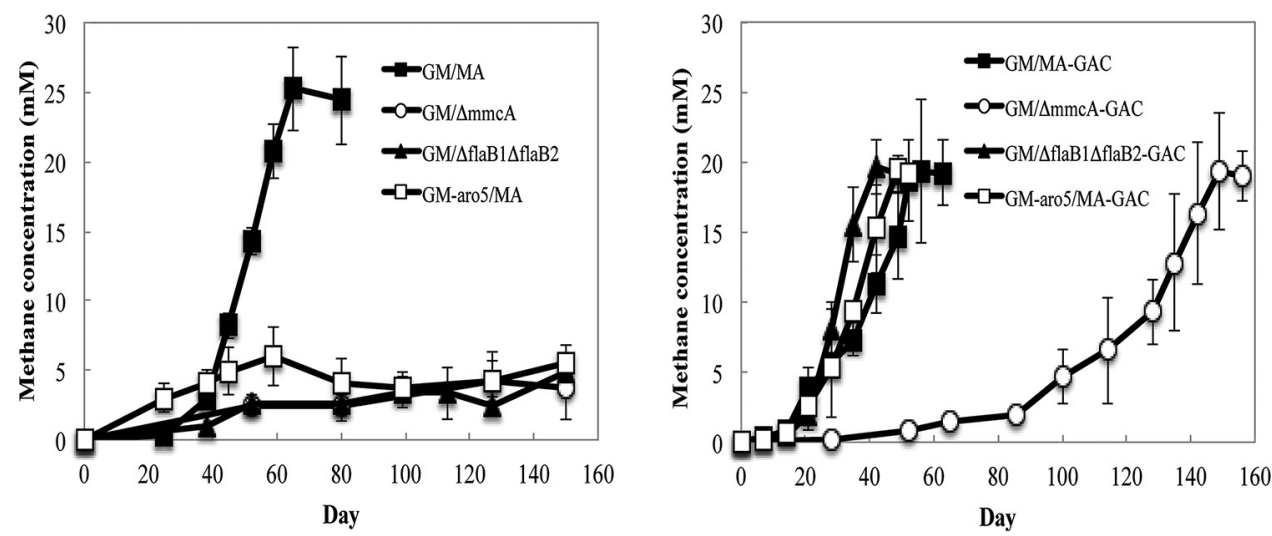

FIG 4 Methane production during initial establishment of cocultures with $G$. metallireducens and various $M$. acetivorans strains grown with ethanol $(20 \mathrm{mM})$ provided as the electron donor in the absence (a) or presence (b) of granular activated carbon (GAC). Data are means and standard deviations for triplicate cultures. GM, wildtype G. metallireducens; GM-Aro5, the Aro-5 strain of G. metallireducens expressing poorly conductive pili; MA, wild-type $M$. acetivorans; $\triangle m m c A, M$. acetivorans strain lacking the gene for the multiheme cytochrome MmcA; $\Delta$ flaB1 $\triangle$ flaB2, M. acetivorans strain lacking the genes for the archaellins FlaB1 and FlaB2.

of the two species often appeared to be in direct contact (Fig. $3 \mathrm{c}$ and d). However, there were instances in which filaments (diameter, ca. $15 \mathrm{~nm}$ ), consistent with the appearance of the $M$. acetivorans archaellum (33), appeared to emanate from M. acetivorans and connect to juxtaposed cells of $G$. metallireducens (Fig. 3e and f).

Genes coding for archaellum proteins were not more significantly expressed in DIET- than acetate-grown cells (Table S1), as might be expected, because M. acetivorans also expresses archaella during growth on acetate (33). In order to evaluate whether the M. acetivorans archaella might play a role in DIET, genes for putative archaellin proteins, FlaB1 and FlaB2, were deleted, yielding a strain that did not express archaella (Fig. S1d). The archaellum-deficient strain did not form an effective DIET coculture with $G$. metallireducens for over 150 days (Fig. 4a). However, when the cocultures were amended with granular activated carbon (GAC), the cocultures initiated with the archaellum-deficient strain produced methane as effectively as cocultures initiated with the parent M. acetivorans strain, which expressed archaella (Fig. 4b).

GAC and other electrically conductive carbon materials can stimulate DIET between wild-type partners and can enable DIET when genes for key extracellular electron transport proteins that are otherwise essential for DIET, like e-pili, have been deleted (34-37). The DIET partners attach to the GAC rather than each other, and the GAC serves as the conduit for long-range interspecies electron transfer (2). Archaella are homologous to type IV pili (38), and the archaellum of Methanospirillum hungatei has a conductance 4-fold higher than that of $G$. sulfurreducens e-pili, demonstrating that at least some archaella can be electrically conductive and might have the potential to be involved in cellto-cell electron transfer (39). The conductivities of a variety of e-pili as well as the Ms. hungatei archaellum are associated with a high abundance of aromatic amino acids $(>9 \%)$ in the pilin/archaellin monomers and no large regions ( $>40$ amino acids) without aromatic amino acids (18). The high density of aromatic amino acids (FlaB1, 11.3\%; FlaB2, 9.5\%) and the lack of large aromatic-free gaps (largest gaps: FlaB1, 26 amino acids; FlaB2, 29 amino acids) in the M. acetivorans archaellins suggest that they might yield conductive archaella. The ability of GAC to rescue the archaellum-deficient strain to enable DIET is consistent with a possible role for archaella in long-range electron transport. However, other, more traditional roles of archaella, such as conferring motility and facilitating attachment (40), might also help cells locate a DIET partner and/or establish initial interspecies contact. In order to more definitively evaluate a role for the M. acetivorans archaellum in interspecies electron transfer, it will be necessary to follow the approach employed for evaluating the role of Geobacter e-pili in DIET (37) and construct a strain 
that expresses an archaellum of with potentially low conductivity. However, such studies are technically difficult and well beyond the scope of the current investigation.

Previous studies have suggested that $G$. metallireducens must express e-pili in order to establish DIET with some $(16,31)$, but not all $(20)$, methanogens. Cocultures initiated with $M$. acetivorans and the previously described (37) G. metallireducens strain Aro-5, which expresses poorly conductive pili (37), did not produce methane for more than 9 months but were not impaired when GAC was added (Fig. 4). This finding is consistent with the previously suggested significance of $G$. metallireducens e-pili for establishment of DIET with M. barkeri (16).

Role for the outer-surface cytochrome MmcA. Gene expression and deletion studies have indicated that the outer surface multiheme c-type cytochrome MmcA is an important component in $M$. acetivorans for electron transfer to the extracellular electron acceptor AQDS (29). Gene transcripts for MmcA were 5 -fold higher $(P=0.008)$ during growth via DIET than growth on acetate (Fig. 2; Table S1). Methane production was inhibited in cocultures initiated with an MmcA-deficient strain of M. acetivorans for over 150 days (Fig. 4a). These results suggest that MmcA may provide an important route for extracellular electron exchange during DIET.

Unlike the archaellum-deficient mutant, GAC did not rapidly rescue the growth of the MmcA-deficient mutant in coculture (Fig. 4b). The cocultures initiated with the MmcA-deficient strain grew only after a very long lag period. The poor methane production even in the presence of GAC is consistent with the fact that MmcA is thought to be embedded in the membrane of $M$. acetivorans $(25,41)$. Thus, its role in extracellular electron transfer is expected to be facilitating transmembrane electron transport. Although GAC can enhance long-range electron exchange between the outer cell surfaces of different species, it does not have a conceivable role in electron transfer across the cell membrane.

$M$. acetivorans has genes for four other putative c-type cytochromes, but the presence of these proteins in $M$. acetivorans has yet to be verified, and deletion of the genes for each of the four cytochrome genes had no impact on extracellular electron transfer to AQDS, even though several of the genes had higher transcript abundance when grown with AQDS as the electron acceptor (29). Transcript abundance for three of these putative cytochrome genes (MA0167, MA2925, and MA3739) was higher in DIET-grown M. acetivorans than in acetate-grown cells (Table S1). Further studies to attempt to document the expression of these proteins in $M$. acetivorans are warranted.

Potential role for Rnf and Fpo complexes in DIET. MmcA has the potential to exchange electrons with methanophenazine or the membrane-bound Rnf complex RnfCDGEAB (25, 42-44). Methanophenazine is an important membrane-bound electron carrier, and the Rnf complex is physically associated with MmcA in the M. acetivorans membrane $(25,41)$. The Rnf complex oxidizes reduced ferredoxin with concomitant transport of sodium across the cell membrane from the cell interior to exterior $(25,42)$. It is proposed that the electrons from ferredoxin oxidation are transferred directly to methanophenazine during acetotrophic methanogenesis $(25,42,45)$ or to MmcA during reduction of extracellular electron acceptors such as Fe(III) and $\operatorname{AQDS}(25,29,46)$.

Transcripts for the majority of genes coding for Rnf subunits were more significantly expressed in DIET-grown cells than acetate-grown cells (Fig. 2; Table S2), suggesting an enhanced role for the Rnf complex during DIET. It seems possible that during DIET, the Rnf complex functions in the reverse direction relative to that proposed for extracellular electron transfer, i.e., accepting electrons to generate the reduced ferredoxin that is required for the first step in the reduction of carbon dioxide to methane (Fig. 2). The most likely electron donor to the Rnf complex is MmcA, which is thought to exchange electrons with Rnf in other forms of M. acetivorans electron transfer (25) and, as noted above, is important for DIET (Fig. 2). The ferredoxin reduction requires transfer of sodium to the interior of the cell via the Rnf complex (Fig. 2). Ten genes coding for ferredoxin proteins and a gene coding for an unusual flavodoxin (FIdA) that can replace ferredoxin as an electron donor under iron-limiting conditions (47) were $>2$-fold more highly expressed $(P<0.05)$ in DIET-grown cells (Table S1). 
Although the pathway for the biosynthesis of methanophenazine has not been deciphered yet, it resembles respiratory quinones in that it has a polyprenyl side chain connected to a redox-active moiety $(48,49)$. Geranylfarnesyl diphosphate is a biosynthetic precursor of methanophenazine, and a homolog (MA0606) of the geranylfarnesyl diphosphate synthase (MM0789) required for methanophenazine biosynthesis in M. mazei (50) was 2.42-fold $(P=0.01)$ more highly expressed in DIET-grown cells (Table S1).

The pathway for carbon dioxide reduction to methane also requires reduced $F_{420}$ (51). The membrane-bound $F_{420}$ dehydrogenase of $M$. barkeri can accept electrons from reduced methanophenazine to generate reduced $F_{420}(52)$ and has been proposed to catalyze $F_{420}$ reduction in a similar manner during $M$. barkeri DIET-based growth (21). This reaction requires concomitant proton translocation from the outside of the cell to the cell interior. Genes for all but one of the Fpo subunits were more highly expressed during $M$. acetivorans growth via DIET than growth on acetate (Fig. 2; Table S3). Therefore, electron transfer from MmcA to methanophenazine followed by electron transfer to Fpo is a likely route for generating $\mathrm{F}_{420} \mathrm{H}_{2}$ to support carbon dioxide reduction during DIET (Fig. 2).

As in other methanogens, methane production in $M$. acetivorans also requires an electron donor to reduce coenzyme M 7-mercaptoheptanoylthreonine-phosphate heterodisulfide (COMS-SCOB) to regenerate coenzyme $\mathrm{M}$ (25). It is proposed that during acetoclastic growth the membrane-bound HdrED complex accepts electrons from methanophenazine reduced by the Rnf complex to reduce COMS-SCOB to HSCOM and $\mathrm{HSCOB}$ while pumping two protons from the interior of the cell across the cell membrane (25). Even though the HdrED complex is required for the conversion of acetate to methane, genes for components of this complex were more highly expressed during growth via DIET (Fig. 2; Table S4). Thus, HdrED is a likely catalyst for CoMS-SCoB reduction (Fig. 2). An alternative strategy for reducing CoMS-SCOB is for HdrABC complexes to oxidize $\mathrm{F}_{420} \mathrm{H}_{2}$ in an electron bifurcation reaction that reduces both ferredoxin and CoMS-SCOB $(53,54)$. Genes for components of the $M$. acetivorans HdrABC complexes were more highly expressed in DIET-grown cells, suggesting the possibility for multiple routes for electron flux during DIET (Table S4).

The proposed route for electron flux during DIET (Fig. 2) demonstrates the possibility for energy conservation from carbon dioxide reduction to methane with electrons derived from DIET. The oxidation of two ethanols to acetate and carbon dioxide yields eight electrons required to reduce carbon dioxide to methane. The eight protons that are also generated from this ethanol metabolism must be consumed in order to prevent acidification within the DIET aggregates. Half of these protons are consumed with the proposed Fpo generation of $\mathrm{F}_{420} \mathrm{H}_{2}$ (Fig. 2). External sodium ions are needed for the proposed Rnf generation of reduced ferredoxin. This requirement can be met by the $\mathrm{H}^{+} / \mathrm{Na}^{+}$antiporter complex (MrpABCDEFG), which adjusts the $\mathrm{H}^{+} / \mathrm{Na}^{+}$ratio for optimal ATP synthesis by $A_{1} A_{0}$ ATP synthase $(55,56)$. As might be expected, genes for components of this complex are more highly expressed in DIET-grown cells (Fig. 2; Table S2). The proposed consumption of 10 positive charges in the reactions catalyzed by the Fpo and Rnf complexes consumes two more positive charges than the eight that are available from ethanol metabolism. However, the export of two sodiums during the reaction catalyzed by the MtrA-H complex and the two protons exported by HdrED, as well as protons consumed in intracellular reactions, yields a net exterior proton gradient to support ATP generation via ATPase. Detailed functional studies would be required to completely validate this model, but the model is based on previously proposed functions of these $M$. acetivorans components, supporting its feasibility.

Implications. The results demonstrate that $M$. acetivorans can serve as an electronaccepting partner for DIET and reveal potential outer-surface electrical contacts and routes for electron flux to support DIET-driven carbon dioxide reduction. This is significant because $M$. acetivorans, which is genetically tractable and one of the most intensively studied methanogens (25), is an excellent physiological model for the type II 
Methanosarcina species that are abundant in many methanogenic soils, sediments, and subsurface environments (22). The results also suggest that different genera of methanogens are likely to employ different strategies for electron uptake during growth via DIET. For example, although MmcA appears to be important for M. acetivorans DIET, some Methanothrix (31) and Methanobacterium (20) species can participate in DIET but lack c-type cytochromes (24).

DIET mechanisms in $M$. acetivorans also appear to differ significantly from those described in $M$. barkeri (21). This is consistent with other substantial differences between type I (i.e., M. barkeri) and type II (i.e., M. acetivorans) Methanosarcina species (22). M. barkeri lacks MmcA and other c-type cytochromes (24). The lack of an Rnf complex in $M$. barkeri requires that electron transport through the membrane to generate reducing equivalents for carbon dioxide reduction rely on the Fpo complex (21).

The diversity of mechanisms for DIET in methanogens suggests that the strategies that rely on gene expression patterns to evaluate the importance of DIET in methanogenic systems will need to accommodate these differences. The mechanisms for extracellular electron exchange in the bacteria and archaea that predominate in anaerobic environments such as soils, sediments, anaerobic digesters, and intestinal systems are still poorly understood (2). For example, although multiple lines of evidence suggest that e-pili are important for extracellular electron transfer in some Geobacter species, a model for how e-pili interact with the rest of the Geobacter electron transport chain, which could aid in understanding how the archaellum of M. acetivorans might "plug in" to membrane electron transport components during DIET, is not yet available (2). However, the genetic tractability of $M$. acetivorans and the growing information on the biochemistry and function of its key proteins (25), as well as its ability to grow as either an electrogen (transporting electrons to extracellular electron acceptors) $(29,46)$ or an electrotroph (consuming electrons from an external source), as shown here, suggest that $M$. acetivorans is an excellent model microbe for further study of extracellular electron exchange in archaea.

\section{MATERIALS AND METHODS}

Parental strain adaptation for coculture at a compatible salinity. Geobacter metallireducens (ATCC 53774) was routinely cultured at $30^{\circ} \mathrm{C}$ under anaerobic conditions $\left(\mathrm{N}_{2}-\mathrm{CO}_{2}\right.$ ratio, 80:20 [vol/vol]) with ethanol $(20 \mathrm{mM})$ provided as the electron donor and Fe(III) citrate $(56 \mathrm{mM})$ as the electron acceptor in freshwater medium as previously described (57). M. acetivorans strain WWM1 ( $(\mathrm{h} p t)$ (58) (a gift from William Metcalf at the University of Illinois) was routinely cultured at $37^{\circ} \mathrm{C}$ in $\mathrm{HS}$-methanol-acetate medium under strict anaerobic conditions as previously described $(27,59)$.

In order to obtain strains of both microbes that grew at compatible temperatures and salinities, both cultures were adapted to grow at $30^{\circ} \mathrm{C}$ in MA medium, which consisted of the following components per liter: $0.35 \mathrm{~g} \mathrm{~K}_{2} \mathrm{HPO}_{4}, 0.23 \mathrm{~g} \mathrm{KH}_{2} \mathrm{PO}_{4}, 0.5 \mathrm{~g} \mathrm{NH}_{4} \mathrm{Cl}, 4 \mathrm{~g} \mathrm{NaCl}, 1 \mathrm{ml} 0.2 \%$ (wt/vol) $\mathrm{FeSO}_{4} \cdot 7 \mathrm{H}_{2} \mathrm{O}$ (in $0.1 \mathrm{~N} \mathrm{H}_{2} \mathrm{SO}_{4}$ ), $1 \mathrm{ml}$ trace element solution SL-10 (DSMZ, medium 320), $10 \mathrm{mM} \mathrm{NaHCO}, 10 \mathrm{ml}$ Wolin's vitamin solution (DSMZ, medium 141), $0.3 \mathrm{mM}$ L-cysteine- $\mathrm{HCl}, 1 \mathrm{ml} 2.7 \% \mathrm{CaCl}_{2} \cdot 2 \mathrm{H}_{2} \mathrm{O}$, and $1 \mathrm{ml} 4.5 \%$ $\mathrm{MgSO}_{4} \cdot 7 \mathrm{H}_{2} \mathrm{O}$. The sodium bicarbonate, Wolin's vitamins, L-cysteine, $\mathrm{CaCl}_{2}$, and $\mathrm{MgSO}_{4}$ solutions were added from sterile anoxic stocks after the base medium was autoclaved.

For coculture experiments, G. metallireducens and M. acetivorans were grown with $20 \mathrm{mM}$ ethanol provided as the electron donor and carbon dioxide as the electron acceptor at $30^{\circ} \mathrm{C}$ in MA medium as previously described $(16,21)$. For comparative transcriptomic studies, $M$. acetivorans was also grown in MA medium with acetate $(40 \mathrm{mM})$ as the sole electron donor.

M. acetivorans mutants. A mutant strain in which the gene for the multiheme c-type cytochrome MmcA was deleted was described previously (29). Previous studies demonstrated that deletion of $m m c A$ did not prevent the expression of downstream genes (29). A strain in which the genes for the archaellin monomer proteins FlaB1 and FlaB2 were deleted was constructed by replacing flaB1 and flaB2 genes with pac (puromycin resistance gene) (Fig. S1). The upstream and downstream regions of flaB1 and flaB2 were amplified by PCR with the primer pairs TCTCTCGAGTTCCTTGAAGATATTAAAGGTC/TCTAAGC TTAATGAATCACCTCAATATTGTG and TCTGGATCCAGCTTGAAATCAAACCAC/TCTGCGGCCGCCACTGCAG CTATAACAC, respectively. The DNA fragments of the upstream and downstream regions were digested with Xhol/Hindlll and BamHI/Notl, respectively. The upstream fragment was ligated with pJK3 (27), and then the downstream fragment was ligated with the pJK3 containing the upstream fragment. The constructed plasmid was linearized with Xhol, and the linearized plasmid was used for transformation. The deletion of $f l a B 1$ and $f l a B 2$ was verified by PCR with the primer pairs TCTCTCGAGTTCCTTGAAGATAT TAAAGGTC (P1)/CCGCCTGCAGTATTCGTTAC (P3) and ACTCTATGCTTGCAGCTGAC (P4)/TCTGCGGCCGCC ACTGCAGCTATAACAC (P2) (Fig. S1). The replacement with the pac gene was verified by PCR with the primer pair AGAGACCCTATCTTACCTGC (P5)/TCTGCGGCCGCCACTGCAGCTATAACAC (P2) (Fig. S1). The 
absence of flagella in the deletion mutant strain was confirmed with transmission electron microscopy (Fig. S1).

Analytical techniques. Ethanol in solution was monitored with a gas chromatograph equipped with a headspace sampler and a flame ionization detector (Clarus 600; PerkinElmer Inc., CA). Methane in the headspace was measured by gas chromatography with a flame ionization detector (Shimadzu, GC$8 \mathrm{~A}$ ) as previously described (60). Acetate concentrations were measured with a Shimadzu high-performance liquid chromatograph (HPLC) with an Aminex HPX-87H ion exclusion column (300 mm by $7.8 \mathrm{~mm}$ ) and an eluent of $8.0 \mathrm{mM}$ sulfuric acid.

Microscopy. Cells were routinely examined by phase-contrast and fluorescence microscopy (BV-2A filter set) with a Nikon E600 microscope. For transmission electron microscopy (TEM), $7 \mu$ l of cells was drop-cast on plasma-sterilized carbon coated 400 mesh copper ultralight grids for 10 min. Liquid was wicked off, and the grid was stained with $3 \mu \mathrm{l} 2 \%$ uranyl acetate for 15 to $20 \mathrm{~s}$ before the liquid was wicked off and air dried. Transmission electron microscopy was done on an FEl Tecnai 12 microscope at $120 \mathrm{kV}$ (spot size, 3), with a camera exposure of $200 \mathrm{~ms}$.

Cells for confocal microscopy were harvested $(1 \mathrm{ml})$ and vacuumed gently onto a $0.2-\mu \mathrm{m}$ polycarbonate filter, washed with $1 \mathrm{ml}$ of wash buffer for $10 \mathrm{~min}$, vacuumed, stained with the Live/Dead BacLight bacterial viability kit (Thermo Fisher) ( $1 \mathrm{ml}$ staining; $3 \mu \mathrm{l}$ each stain per $\mathrm{ml}$ ) for $10 \mathrm{~min}$, vacuumed, destained with wash buffer for $10 \mathrm{~min}$, vacuumed, and mounted on glass slides with coverslips and antifade reagent in glycerol. Cells were visualized on a Nikon A1R-SIMe confocal microscope.

DNA extraction and quantitative PCR. Genomic DNA was extracted from cocultures with the MasterPure complete DNA purification kit (Lucigen). The proportion of G. metallireducens and M. acetivorans cells in cocultures was determined with quantitative PCR using the following primer sets: (i) GS15-16Sq-f (5'-CAGCTCGTGTCGTGAGATGT-3') and GS15-16Sq-r (5'-GTTGACACCGGCAGTTCT-3'), which amplified a 106-bp fragment from the 16S rRNA gene of $G$. metallireducens, and (ii) MA-16Sq-f (5'GTAGTCCCAGCCGTAAACGA-3') and MA-16Sq-r (5'-CCCGCCAATCCTTAAGTT-3'), which amplified a 132-bp fragment of the $M$. acetivorans 16S rRNA gene. Both G. metallireducens and M. acetivorans have three copies of the 16S rRNA gene in their genomes. Therefore, qPCR results were not influenced by unequal gene copy numbers. Standard curve analysis of both primer sets revealed that they had $>95 \%$ efficiencies, and melting curve analysis yielded a single peak, indicating that they were highly specific.

Power SYBR green PCRm master mix (Applied Biosystems, Foster City, CA) and an ABI 7500 real-time PCR system were used to amplify and to quantify all PCR products. Each reaction mixture (25 $\mu \mathrm{l})$ consisted of forward and reverse primers at a final concentration of $200 \mathrm{nM}, 5 \mathrm{ng}$ of gDNA, and $12.5 \mu \mathrm{l}$ of Power SYBR green PCR master mix (Applied Biosystems).

RNA extraction. Cells were harvested from triplicate $50-\mathrm{ml}$ cultures of $M$. acetivorans grown alone with acetate $(40 \mathrm{mM})$ provided as a substrate (acetate conditions), or $50 \mathrm{ml}$ cultures of M. acetivorans grown in coculture with G. metallireducens with ethanol $(20 \mathrm{mM})$ provided as an electron donor (DIET condition). Cells were harvested during mid-exponential phase when $\sim 18 \mathrm{mM}$ methane was detected in the headspace.

Cells were split into 50-ml conical tubes (BD Sciences), mixed with RNA Protect (Qiagen) in a 1:1 ratio, and pelleted by centrifugation at $3,000 \times g$ for $15 \mathrm{~min}$ at $4^{\circ} \mathrm{C}$. Pellets were then immediately frozen in liquid nitrogen and stored at $-80^{\circ} \mathrm{C}$. Total RNA was extracted from cell pellets as previously described (61), and all six RNA samples ( 3 acetate, 3 DIET) were cleaned with the RNeasy minikit (Qiagen) and treated with Turbo DNA-free DNase (Ambion). PCR with primers targeting the 16S rRNA gene was then done on all samples to ensure that they were not contaminated with genomic DNA. mRNA was then further enriched from all samples with the MicrobExpress kit (Ambion), according to the manufacturer's instructions.

Illumina sequencing and data analysis. The ScriptSeq v2 RNA-Seq library preparation kit (Epicentre) was used to prepare directional multiplex libraries. Paired-end sequencing was then performed on these libraries with a Hi-Seq 2000 platform at the Deep Sequencing Core Facility at the University of Massachusetts Medical School in Worchester, MA.

Raw data were quality checked with FASTQC (http://www.bioinformatics.babraham.ac.uk/projects/ fastqc/), and initial raw nonfiltered forward and reverse sequencing libraries contained an average of $68,911,030 \pm 21,863,730$ reads that were $\sim 100$ bp long (Table S5). Sequences from all of the libraries were trimmed and filtered with Trimmomatic (62), which yielded an average of 55,239,290 $\pm 29,060,121$ quality reads per transcriptome sequencing (RNA-seq) library.

All paired-end reads were then merged with FLASH (63), resulting in 32,159,242 $\pm 22,219,390$ reads with an average read length of $134 \pm 28 \mathrm{bp}$. rRNA reads were then removed from the libraries with SortMeRNA (64), which resulted in 4,959,312 $\pm 2,340,361$ mRNA reads.

Mapping of $\mathbf{m R N A}$ reads. Trimmed and filtered $m R N A$ reads from the triplicate samples for the two different culture conditions were mapped against the M. acetivorans strain C2A genome (NC_003552) downloaded from IMG/MER (img.jgi.doe.gov) using ArrayStar software (DNAStar). Reads were normalized and processed for differential expression studies using the edgeR package in Bioconductor (65). All genes that were $\geq 2$-fold differentially expressed with $P$ values of $\leq 0.05$ are reported in Table S1.

Data availability. Illumina sequence reads have been submitted to the SRA NCBI database under BioProject number PRJNA727272 and Biosample numbers SAMN19011637 and SAMN19011638.

\section{SUPPLEMENTAL MATERIAL}

Supplemental material is available online only.

FIG S1, TIF file, 1.5 MB.

TABLE S1, XLSX file, $0.2 \mathrm{MB}$. 
TABLE S2, DOCX file, $0.1 \mathrm{MB}$.

TABLE S3, DOCX file, $0.1 \mathrm{MB}$.

TABLE S4, DOCX file, $0.1 \mathrm{MB}$.

TABLE S5, DOCX file, $0.1 \mathrm{MB}$.

\section{ACKNOWLEDGMENTS}

The confocal microscopy data were gathered in the Light Microscopy Facility and Nikon Center of Excellence at the Institute for Applied Life Sciences, UMass Amherst, with support from the Massachusetts Life Sciences Center. The transmission electron microscopy images were collected in the Electron Microscopy Facility at the Institute for Applied Life Sciences, UMass Amherst.

This research was supported by the Army Research Office and was accomplished under grant W911NF-17-1-0345. The views and conclusions contained in this document are those of the authors and should not be interpreted as representing the official policies, either expressed or implied, of the Army Research Office or the U.S. Government.

We do not declare any conflicts of interest.

\section{REFERENCES}

1. Whiticar M. 2020. The biogeochemical methane cycle, p 669-746. In Wilkes $\mathrm{H}$ (ed), Hydrocarbons, oils and lipids: diversity, origin, chemistry and fate. Handbook of hydrocarbons and lipid microbiology. Springer $\mathrm{Na}$ ture, Cham, Switzerland.

2. Lovley DR, Holmes DE. 2021. Electromicrobiology: the ecophysiology of phylogenetically diverse electroactive microorganisms. Nat Rev Microbiol https://doi.org/10.1038/s41579-021-00597-6. Epub ahead of print.

3. Lovley DR. 2017. Syntrophy goes electric: direct interspecies electron transfer. Annu Rev Microbiol 71:643-664. https://doi.org/10.1146/annurev-micro -030117-020420.

4. Zhao Z, Li Y, Zhang Y, Lovley D. 2020. Sparking anaerobic digestion: promoting direct interspecies electron transfer to enhance methane production. iScience 23:101794. https://doi.org/10.1016/j.isci.2020.101794.

5. Rotaru AE, Yee MO, Musat F. 2021. Microbes trading electricity in consortia of environmental and biotechnological significance. Curr Opin Biotechnol 67:119-129. https://doi.org/10.1016/j.copbio.2021.01.014.

6. Stams AJ, Plugge CM. 2009. Electron transfer in syntrophic communities of anaerobic bacteria and archaea. Nat Rev Microbiol 7:568-577. https:// doi.org/10.1038/nrmicro2166.

7. Mclnerney MJ, Struchtemeyer CG, Sieber J, Mouttaki H, Stams AJ, Schink B, Rohlin L, Gunsalus RP. 2008. Physiology, ecology, phylogeny, and genomics of microorganisms capable of syntrophic metabolism. Ann N Y Acad Sci 1125:58-72. https://doi.org/10.1196/annals.1419.005.

8. Sieber JR, Mclnerney MJ, Gunsalus RP. 2012. Genomic insights into syntrophy: the paradigm for anaerobic metabolic cooperation. Annu Rev Microbiol 66:429-452. https://doi.org/10.1146/annurev-micro-090110-102844.

9. Sieber JR, Le HM, Mclnerney MJ. 2014. The importance of hydrogen and formate transfer for syntrophic fatty, aromatic and alicyclic metabolism. Environ Microbiol 16:177-188. https://doi.org/10.1111/1462-2920.12269.

10. Rotaru AE, Shrestha PM, Liu FH, Ueki T, Nevin K, Summers ZM, Lovley DR. 2012. Interspecies electron transfer via hydrogen and formate rather than direct electrical connections in cocultures of Pelobacter carbinolicus and Geobacter sulfurreducens. Appl Environ Microbiol 78:7645-7651. https:// doi.org/10.1128/AEM.01946-12.

11. Conrad R, Phelps TJ, Zeikus JG. 1985. Gas metabolism evidence in support of the juxtaposition of hydrogen-producing and methanogenic bacteria in sewage sludge and lake sediments. Appl Environ Microbiol 50:595-601 . https://doi.org/10.1128/aem.50.3.595-601.1985.

12. Conrad R, Babbel M. 1989. Effect of dilution on methanogenesis, hydrogen turnover and interspecies hydrogen transfer in anoxic paddy soil. FEMS Microbiol Ecol 62:21-28. https://doi.org/10.1111/j.1574-6968.1989 .tb03654.x.

13. Conrad R, Mayer H-P, WüSt M. 1989. Temporal change of gas metabolism by hydrogen-syntrophic methanogenic bacterial associations in anoxic paddy soil. FEMS Microbiol Ecol 62:265-273. https://doi.org/10.1111/j .1574-6968.1989.tb03701.x.

14. Lovley D, Klug M. 1982. Intermediary metabolism of organic carbon in the sediment of a eutrophic lake. Appl Environ Microbiol 43:552-560. https:// doi.org/10.1128/aem.43.3.552-560.1982.
15. Morita M, Malvankar NS, Franks AE, Summers ZM, Giloteaux L, Rotaru AE, Rotaru C, Lovley DR. 2011. Potential for direct interspecies electron transfer in methanogenic wastewater digester aggregates. mBio 2:e00159-11. https://doi.org/10.1128/mBio.00159-11.

16. Rotaru AE, Shrestha PM, Liu F, Markovaite B, Chen S, Nevin KP, Lovley DR 2014. Direct interspecies electron transfer between Geobacter metallireducens and Methanosarcina barkeri. Appl Environ Microbiol 80:4599-4605 . https://doi.org/10.1128/AEM.00895-14.

17. Holmes DE, Shrestha PM, Walker DJF, Dang Y, Nevin KP, Woodard TL, Lovley DR. 2017. Metatranscriptomic evidence for direct interspecies electron transfer between Geobacter and Methanothrix species in methanogenic rice paddy soils. Appl Environ Microbiol 83:e00223-17. https://doi .org/10.1128/AEM.00223-17.

18. Walker DJF, Nevin KP, Holmes DE, Rotaru AE, Ward JE, Woodard TL, Zhu J, Ueki T, Nonnenmann SS, Mclnerney MJ, Lovley DR. 2020. Syntrophus conductive pili demonstrate that common hydrogen-donating syntrophs can have a direct electron transfer option. ISME J 14:837-846. https://doi.org/ 10.1038/s41396-019-0575-9.

19. Yee MO, Rotaru AE. 2020. Extracellular electron uptake in Methanosarcinales is independent of multiheme c-type cytochromes. Sci Rep 10:372. https://doi.org/10.1038/s41598-019-57206-z.

20. Zheng S, Liu F, Wang B, Zhang Y, Lovley DR. 2020. Methanobacterium capable of direct interspecies electron transfer. Environ Sci Technol 54: 15347-15354. https://doi.org/10.1021/acs.est.0c05525.

21. Holmes DE, Rotaru AE, Ueki T, Shrestha PM, Ferry JG, Lovley DR. 2018 Electron and proton flux for carbon dioxide reduction in Methanosarcina barkeri during direct interspecies electron transfer. Front Microbiol 9: 3109. https://doi.org/10.3389/fmicb.2018.03109.

22. Zhou J, Holmes D, Tang H, Lovley D. 2021. Correlation of key physiological properties of Methanosarcina isolates with environment of origin. Appl Environ Microbiol 87:e00731-21. https://doi.org/10.1128/AEM.00731-21.

23. Mo Y, Snoeyenbos-West OL, Thamdrup B, Ottosen LDM, Rotaru AE. 2019. Extracellular electron uptake by two Methanosarcina species. Front Energy Res 7:29.

24. Kletzin A, Heimerl T, Flechsler J, van Niftrik L, Rachel R, Klingl A. 2015. Cytochromes $c$ in Archaea: distribution, maturation, cell architecture, and the special case of Ignicoccus hospitalis. Front Microbiol 6:439. https://doi .org/10.3389/fmicb.2015.00439.

25. Ferry JG. 2020. Methanosarcina acetivorans: a model for mechanistic understanding of aceticlastic and reverse methanogenesis. Front Microbiol 11:1806. https://doi.org/10.3389/fmicb.2020.01806.

26. Buan N, Kulkarni G, Metcalf W. 2011. Genetic methods for Methanosarcina species. Methods Enzymol 494:23-42. https://doi.org/10.1016/B978-0-12 -385112-3.00002-0.

27. Metcalf WW, Zhang JK, Apolinario E, Sowers KR, Wolfe RS. 1997. A genetic system for Archaea of the genus Methanosarcina: liposome-mediated transformation and construction of shuttle vectors. Proc Natl Acad Sci U S A 94:2626-2631. https://doi.org/10.1073/pnas.94.6.2626. 
28. Nayak DD, Metcalf WW. 2017. Cas9-mediated genome editing in the methanogenic archaeon Methanosarcina acetivorans. Proc Natl Acad Sci U S A 114:2976-2981. https://doi.org/10.1073/pnas.1618596114.

29. Holmes DE, Ueki T, Tang HY, Zhou J, Smith JA, Chaput G, Lovley DR. 2019. A membrane-bound cytochrome enables Methanosarcina acetivorans to conserve energy from extracellular electron transfer. mBio 10:e00789-19. https://doi.org/10.1128/mBio.00789-19.

30. Summers ZM, Fogarty HE, Leang C, Franks AE, Malvankar NS, Lovley DR. 2010. Direct exchange of electrons within aggregates of an evolved syntrophic coculture of anaerobic bacteria. Science 330:1413-1415. https:// doi.org/10.1126/science.1196526.

31. Rotaru AE, Shrestha PM, Liu FH, Shrestha M, Shrestha D, Embree M, Zengler K, Wardman C, Nevin KP, Lovley DR. 2014. A new model for electron flow during anaerobic digestion: direct interspecies electron transfer to Methanosaeta for the reduction of carbon dioxide to methane. Energy Environ Sci 7:408-415. https://doi.org/10.1039/C3EE42189A.

32. Shrestha PM, Rotaru AE, Summers ZM, Shrestha M, Liu F, Lovley DR. 2013. Transcriptomic and genetic analysis of direct interspecies electron transfer. Appl Environ Microbiol 79:2397-2404. https://doi.org/10.1128/AEM .03837-12.

33. Sowers KR, Baron SF, Ferry JG. 1984. Methanosarcina acetivorans sp. nov., an acetotrophic methane-producing bacterium isolated from marine sediments. Appl Environ Microbiol 47:971-978. https://doi.org/10.1128/ aem.47.5.971-978.1984.

34. Chen S, Rotaru AE, Liu F, Philips J, Woodard TL, Nevin KP, Lovley DR. 2014. Carbon cloth stimulates direct interspecies electron transfer in syntrophic co-cultures. Bioresour Technol 173:82-86. https://doi.org/10.1016/j.biortech .2014.09.009.

35. Chen S, Rotaru AE, Shrestha PM, Malvankar NS, Liu F, Fan W, Nevin KP, Lovley DR. 2014. Promoting interspecies electron transfer with biochar. Sci Rep 4:5019. https://doi.org/10.1038/srep05019.

36. Liu F, Rotaru A, Shrestha P, Malvankar N, Nevin K, Lovley D. 2012. Promoting direct interspecies electron transfer with activated carbon. Energy Environ Sci 5:8982-8989. https://doi.org/10.1039/c2ee22459c.

37. Ueki T, Nevin KP, Rotaru A-E, Wang L-Y, Ward JE, Woodard TL, Lovley DR. 2018. Geobacter strains expressing poorly conductive pili reveal constraints on direct interspecies electron transfer mechanisms. mBio 9: e01273-18. https://doi.org/10.1128/mBio.01273-18.

38. Beeby M, Ferreira JL, Tripp P, Albers SV, Mitchell DR. 2020. Propulsive nanomachines: the convergent evolution of archaella, flagella and cilia. FEMS Microbiol Rev 44:253-304. https://doi.org/10.1093/femsre/fuaa006.

39. Walker D, Martz E, Holmes D, Zhou Z, Nonnenmann S, Lovley D. 2019. The archaellum of Methanospirillum hungatei is electrically conductive. mBio 10:e00579-19. https://doi.org/10.1128/mBio.00579-19.

40. Jarrell K, Albers S, de Sousa Machado J. 2021. A comprehensive history of motility and archaellation in Archaea. FEMS Microbes 2:xtab002. https:// doi.org/10.1093/femsmc/xtab002.

41. Li Q, Li L, Rejtar T, Lessner DJ, Karger BL, Ferry JG. 2006. Electron transport in the pathway of acetate conversion to methane in the marine archaeon Methanosarcina acetivorans. J Bacteriol 188:702-710. https://doi.org/10 .1128/JB.188.2.702-710.2006.

42. Schlegel K, Welte C, Deppenmeier U, Muller V. 2012. Electron transport during aceticlastic methanogenesis by Methanosarcina acetivorans involves a sodium-translocating Rnf complex. FEBS J 279:4444-4452. https://doi.org/10.1111/febs.12031.

43. Suharti S, Wang M, de Vries S, Ferry JG. 2014. Characterization of the RnfB and RnfG subunits of the Rnf complex from the archaeon Methanosarcina acetivorans. PLoS One 9:e97966. https://doi.org/10.1371/journal.pone .0097966 .

44. Wang M, Tomb JF, Ferry JG. 2011. Electron transport in acetate-grown Methanosarcina acetivorans. BMC Microbiol 11:165. https://doi.org/10 .1186/1471-2180-11-165.

45. Welte C, Deppenmeier U. 2014. Bioenergetics and anaerobic respiratory chains of aceticlastic methanogens. Biochim Biophys Acta 1837:1130-1147 . https://doi.org/10.1016/j.bbabio.2013.12.002.

46. Prakash D, Chauhan SS, Ferry JG. 2019. Life on the thermodynamic edge: respiratory growth of an acetotrophic methanogen. Sci Adv 5:eaaw9059. https://doi.org/10.1126/sciadv.aaw9059.

47. Prakash D, lyer PR, Suharti S, Walters KA, Santiago-Martinez MG, Golbeck JH, Murakami KS, Ferry JG. 2019. Structure and function of an unusual flavodoxin from the domain Archaea. Proc Natl Acad Sci U S A 116: 25917-25922. https://doi.org/10.1073/pnas.1908578116.

48. Beifuss U, Tietze M, Baumer S, Deppenmeier U. 2000. Methanophenazine: strucrture, total synthesis, and function of a new cofactor from methanogenic archaea. Angew Chem Int Ed 39:2470-2471. https://doi.org/10 .1002/1521-3773(20000717)39:14<2470::AID-ANIE2470>3.0.CO;2-R.

49. Abken HJ, Tietze M, Brodersen J, Baumer S, Beifuss U, Deppenmeier U. 1998. Isolation and characterization of methanophenazine and function of phenazines in membrane-bound electron transport of Methanosarcina mazei Gol. J Bacteriol 180:2027-2032. https://doi.org/10.1128/JB.180.8 .2027-2032.1998.

50. Ogawa T, Yoshimura T, Hemmi H. 2010. Geranylfarnesyl diphosphate synthase from Methanosarcina mazei: different role, different evolution. Biochem Biophys Res Commun 393:16-20. https://doi.org/10.1016/j.bbrc .2010.01.063.

51. Thauer RK, Kaster AK, Seedorf H, Buckel W, Hedderich R. 2008. Methanogenic archaea: ecologically relevant differences in energy conservation. Nat Rev Microbiol 6:579-591. https://doi.org/10.1038/nrmicro1931.

52. Mand T, Kulkarni G, Metcalf W. 2018. Genetic, biochemical, and molecular characterization of Methanosarcina barkeri mutants lacking three distinct classes of hydrogenase. J Bacteriol 200:e00342-18. https://doi.org/10 .1128/JB.00342-18.

53. Yan Z, Ferry J. 2018. Electron bifurcation and confurcation in methanogenesis and reverse methanogenesis. Front Microbiol 9:1322. https://doi .org/10.3389/fmicb.2018.01322.

54. Yan Z, Wang MY, Ferry JG. 2017. A ferredoxin-and F420H2-dependent, electron-bifurcating, heterodisulfide reductase with homologs in the domains bacteria and archaea. mBio 8:e02285-16. https://doi.org/10 $.1128 / \mathrm{mBio} .02285-16$.

55. Jasso-Chavez R, Apolinario EE, Sowers KR, Ferry JG. 2013. MrpA functions in energy conversion during acetate-dependent growth of Methanosarcina acetivorans. J Bacteriol 195:3987-3994. https://doi.org/10.1128/JB .00581-13.

56. Jasso-Chavez R, Diaz-Perez C, Rodriguez-Zavala JS, Ferry JG. 2017. Functional role of MrpA in the MrpABCDEFG Na$/ / \mathrm{H}^{+}$antiporter complex from the archaeon Methanosarcina acetivorans. J Bacteriol 199:e00662-16. https://doi.org/10.1128/JB.00662-16.

57. Lovley DR, Giovannoni SJ, White DC, Champine JE, Phillips EJ, Gorby YA, Goodwin S. 1993. Geobacter metallireducens gen. nov. sp. nov., a microorganism capable of coupling the complete oxidation of organic compounds to the reduction of iron and other metals. Arch Microbiol 159: 336-344. https://doi.org/10.1007/BF00290916.

58. Pritchett MA, Zhang JK, Metcalf WW. 2004. Development of a markerless genetic exchange method for Methanosarcina acetivorans C2A and its use in construction of new genetic tools for methanogenic archaea. Appl Environ Microbiol 70:1425-1433. https://doi.org/10.1128/AEM.70.3.1425 $-1433.2004$.

59. Sowers KR, Boone JE, Gunsalus RP. 1993. Disaggregation of Methanosarcina spp. and growth as single cells at elevated osmolarity. Appl Environ Microbiol 59:3832-3839. https://doi.org/10.1128/aem.59.11.3832-3839 .1993.

60. Holmes DE, Giloteaux L, Orellana R, Williams KH, Robbins MJ, Lovley DR. 2014. Methane production from protozoan endosymbionts following stimulation of microbial metabolism within subsurface sediments. Front Microbiol 5:366. https://doi.org/10.3389/fmicb.2014.00366.

61. Holmes DE, Risso C, Smith JA, Lovley DR. 2012. Genome-scale analysis of anaerobic benzoate and phenol metabolism in the hyperthermophilic archaeon Ferroglobus placidus. ISME J 6:146-157. https://doi.org/10.1038/ ismej.2011.88.

62. Bolger AM, Lohse M, Usadel B. 2014. Trimmomatic: a flexible trimmer for Illumina sequence data. Bioinformatics 30:2114-2120. https://doi.org/10 .1093/bioinformatics/btu170.

63. Magoc T, Salzberg SL. 2011. FLASH: fast length adjustment of short reads to improve genome assemblies. Bioinformatics 27:2957-2963. https://doi .org/10.1093/bioinformatics/btr507.

64. Kopylova E, Noe L, Touzet H. 2012. SortMeRNA: fast and accurate filtering of ribosomal RNAs in metatranscriptomic data. Bioinformatics 28:3211-3217. https://doi.org/10.1093/bioinformatics/bts611.

65. Robinson MD, McCarthy DJ, Smyth GK. 2010. edgeR: a Bioconductor package for differential expression analysis of digital gene expression data. Bioinformatics 26:139-140. https://doi.org/10.1093/bioinformatics/btp616. 